\title{
ARE THERE GHOST IMAGES OF THE COMA CLUSTER AT OTHER REDSHIFTS?
}

\author{
BOUDEWIJN F. ROUKEMA \\ National Observatory of Japan, Mitaka, 181 Tokyo, Japan \\ email: roukema@iap.fr
}

The topology of the Universe is a fundamental property of our Universe according to Friedmann-Lemaître models[3, 9, 7], but has not yet been reliably measured. As pointed out by Sato[12], the Universe may be finite even though flat or negatively curved: $k \leq 0 \nRightarrow$ infinite volume of a hypersurface.

Turner[13] listed different questions of physics which cosmologists must address. A significant detection of non-trivial topology could bring quantum cosmology into the realm of observational astronomy.

Ellis[4] argued that any alternatives to the standard model should make observationally refutable predictions. Topology is usually considered within the standard model (e.g., [6, 5]). Nevertheless, any specific candidate topology makes refutable predictions, given appropriate telescope time. Proof that the candidate ghost images of the Coma cluster at $z \sim 0.45$ [11] are not Coma is in principle straightforward.

However, serious tests for constraining non-trivial topology (e.g. $[8,10$, 2]) require large scale programmes, just as for $\Omega_{0}, \Omega_{b}$ and $H_{0}$.

Burbidge[1] commented about sociology: it is left to the reader to judge whether or not non-trivial topology is politically correct.

\section{References}

1. Burbidge, G., IAU Symposium 183, Panel Discussion on the Standard Cosmology

2. Cornish, N.J., Spergel, D.N., Starkman, G.D., 1997, gr-qc/9602039

3. de Sitter, W., 1917, MNRAS, 78, 3

4. Ellis, R.S., IAU Symposium 183, Panel Discussion on the Standard Cosmology

5. Fagundes, H.V., Wichoski, U.F., 1987, ApJ, 322, L5

6. Gott, J.R.I., 1980, MNRAS, 193, 153

7. Lachièze-Rey, M., Luminet, J.-P., 1995, PhysRep, 254, 136

8. Lehoucq, R., Luminet, J.-P., Lachièze-Rey, M., 1996, A\&A, 313, 339

9. Lemaître, G., 1958, La Struct. et l'Evol. de l'Univers, ed. Stoops, R., Brussels, p1

10. Roukema, B.F., 1996, MNRAS, 283, 1147

11. Roukema, B.F., Edge, A.C., 1997, MNRAS, in press, (astro-ph/9706166)

12. Sato, H., IAU Symposium 183, Panel Discussion on the Standard Cosmology

13. Turner, E., IAU Symposium 183, Panel Discussion on the Standard Cosmology 